\title{
LA UNIVERSIDAD: ¿UN ESCENARIO PARA TRANSFORMAR LA CONCIENCIA SOCIAL DE LOS PROFESIONALES?
}

\author{
Elizabeth Murrain Knudson*
}

\section{Resumen}

En el presente trabajo comparto algunas propuestas que nos permiten reflexionar sobre la misión de la universidad y vindicar el papel que cumple como institución formadora de sujetos con conciencia social, firmes valores humanos que respeten, promuevan y los hagan evidentes en todos y cada uno de sus actos, que no sólo respondan a las necesidades del mundo laboral sino que asuman nuevos retos y jalonen crecimiento, desarrollo y transformación social, política, económica, técnica y científica a nivel local, regional y mundial. Así lo han hecho nuestras universidades durante el siglo XX, permitiendo el avance y desarrollo de Colombia en las urbes, en las regiones e incluso aportando cerebros brillantes a otras naciones. El trabajo desarrolla dos temas: 1) las enseñanzas pedagógicas y epistemológicas de Platón y 2) el analfabetismo científico en que ha caído la formación universitaria. Para finalizar relaciono estos temas con la formación de profesionales de enfermería, cómo se debe trasformar la educación universitaria y la influencia en el actual enfermero para que sea evidente a través del ser, conocer y saber hacer.

Palabras clave: enseñanza universitaria, conciencia social.

\section{IS THE UNIVERSITY A SCENARIO TO TRANSFORM SOCIAL AWARENESS ON PROFESSIONALS?}

\begin{abstract}
In the present work I share some proposals which allow us to meditate on the mission of the university and to recover the role it accomplishes as an institution to prepare subjects with social awareness, sound human values which they respect, encourage and make evident in each and every one of their actions, which not only satisfy the needs of the labor world but also assume new challenges and bring about growth, development and social, political, economic, technical and scientific transformation at local, regional and global levels. This has been achieved by our universities during the 20th century, allowing advance and development of Colombia throughout cities, regions and even has contributed with brilliant minds to other nations.. Two topics are developed in this work: 1) the pedagogic and epistemologic teachings of Plato and 2) the scientific illiteracy in which university education has fallen into. Last, I relate these topics with the training of nursing professionals, how should university education must be transformed and its influence on the current nurse in order to be evident through being, understanding and knowing how.
\end{abstract}

Key words: university education, social awareness.

Fecha recibido: junio 17 de 2008 - Fecha aceptado: septiembre 16 de 2008

* Enfermera egresada de la Universidad Nacional de Colombia, Especialista en Auditoria en Salud, con Diplomado en Epidemiología, dos
Diplomados en Docencia Universitaria, Magistrada del Tribunal Nacional de Ética en Enfermería período 2006 - 2010, Directora de Posgrados Fundación Universitaria Ciencias de la Salud, Bogotá D.C. Candidata al Doctorado en Bioética de la Universidad El Bosque. 
Este nuevo siglo cargado de adversidad, desigualdades que se profundizan, globalización que agudiza las inequidades y grandes amenazas pero con indudables oportunidades, guerras soterradas en donde se evidencia la destrucción implacable de pueblos diferentes y antiguos que están cargados de historia y aportes trascendentales a la humanidad, requiere una revaluación de las universidades, en particular en lo referente a la actitud que asumen los estudiantes y los profesionales en los escenarios académicos y sociales, así como la forma como se desarrollan los procesos educativos que se han preocupado más por brindar títulos que por formar seres con valores e identidades que influyan de manera positiva en el desarrollo de la sociedad.

Resignificación de la formación universitaria que permita generar a partir de ahora esa realidad vital y concreta, formando ciudadanos de Colombia y del mundo sensibles, comprometidos no sólo con su área de influencia y desarrollo profesional, sino que a través de pequeñas acciones transformadoras (conciencia social, masa crítica) empiecen una red utópica quizás pero muy sólida, fuerte e íntegra para generar un cambio social en donde la salud y la educación sean eficientes, oportunas, pertinentes y recobren su sentido de derecho fundamental y no sólo sean la mercancía que se viene promoviendo hace mas de diez años en el país. Como todo producto en el sistema capitalista busca mantenerse a través de la reducción de la inversión para abaratar costos de producción, lo que ha permitido que se pierda de vista la misión y compromiso de la universidad en aras de ser "competentes, eficientes, sostenibles y autofinanciables", con el objeto de ser aceptados con muy buenos ojos por el mercado laboral, como si ésta fuera la máxima de la formación profesional universitaria, ofreciendo al profesional contratos a destajo y con sueldos cada vez más bajos.

\section{Enseñanzas pedagógicas $y$ epistemológicas de Platón}

Recreándonos en los postulados presentados por este filósofo y pedagogo, considero que toman vigencia frente a las características y actitudes que presentan los estudiantes de hoy y el papel que debe desempeñar el docente inquietando, motivando, mostrando el camino de la vida como una fuente inagotable de conocimiento grato y placentero, contribuyendo al logro de los objetivos de la formación tanto humana como científica y técnica, con la competencia de encantar y asombrar al estudiante, no desesperanzar y frustrar...

Es por eso que retomo las enseñanzas pedagógicas y epistemológicas de Platón, creo que si nuestros currículos y pedagogías contemplan esa filosofía podremos abordar de raíz la problemática y fractura entre la universidad y el mercado, la realidad y la utopía, y por lo tanto nuestros estudiantes y egresados además de ser competentes en el conocimiento y la técnica, lo serán en lo referente al desarrollo humano. Estas enseñanzas abordan cinco postulados, los cuales son:

\section{Modestia intelectual}

La ignorancia: "la ignorancia no es un estado de carencia de conocimiento sino de llenura, es un estado de atestamiento intelectual", el individuo cree saber muchas cosas, pero en verdad es mera opinión en la cual se confía sin someterla a la crítica, confrontación, reflexión, análisis y debe promover el respeto, la responsabilidad y la libertad de opinión.

Enseñanza: significa para Platón alimentar, limpiar la mente de opiniones mal fundamentadas, con el fin de que haya espacio para interrogarse y buscar el saber. La conciencia de la propia ignorancia es importante para el desarrollo ético de la actividad intelectual, pedagógica e investigativa. Para esto el maestro debe procurar y promover el respeto y la libertad, ya que debe transmitir que el conocimiento es relativo, falible y circunscrito. Ante lo cual toma sentido la frase de Kart Poper "la ciencia no es la posesión de la verdad, sino su búsqueda c onstante".

\section{El método - la mayéutica}

¿Cómo desarrolla Platón la enseñanza pedagógica y la epistemología fundamental? Expresa el pedagogo y filósofo que es un partero de lo espiritual "él ayuda a dar a luz las ideas, los pensamientos", "no concibe la enseñanza como la mera transmisión de conocimientos, sino como un proceso de descubrimiento y apropiación". 
El proceso de aprendizaje de un saber es individual, donde el alumno va poco a poco construyendo sus categorías conceptuales. "El maestro entiende que él es un compañero en la aventura de sus alumnos hacia la construcción de su propio conocimiento", pasará de una pedagogía transmisionista y repetitiva a una abierta, participativa y dinámica, donde los alumnos son en última los responsables de su propio saber.

Sócrates ayuda a Teeteto a que ofrezca el fruto de su reflexión crítica y argumentación, lo ayuda a desarrollar el conocimiento a través de sus preguntas, pero la mayéutica se limita a secundar la inteligencia que está en "proceso de parto", recoge los frutos, los examina, considera si es viable, los somete a la crítica y genera nuevos esfuerzos.

\section{El asombro}

El conocimiento es fundamentado; la ciencia existe gracias a que otros fueron capaces de asombrarse, maravillarse. El conocimiento se inicia con la admiración "ella impulsó a los primeros pensadores a realizar especulaciones filosóficas." "El comienzo de todos los saberes es la admiración ante el hecho de que las cosas sean lo que son”. Nos pone en condiciones de pensar. El que no se admira ni siquiera puede preguntarse y sin preguntar no hay conocimiento. Plantea la exigencia de conocer la verdad, sólo la genuina admiración despierta la reflexión. Es difícil conquistar un conocimiento fundamentado para quien todo lo ve como evidente y no es capaz de asombrarse, de encontrar algo de misterio en la realidad social o física que lo rodea.

\section{El conocimiento fundamentado}

La apropiación del conocimiento necesita tiempo, no puede ser improvisado, quien lo desee adquirir en cualquier área del saber no puede crear respuestas, necesita tiempo para sacar las consecuencias que van implícitas en las preguntas. La investigación es el único camino para llegar al conocimiento fundamentado y necesita tiempo para desarrollarse. Quien está obligado a dar respuestas en tiempo fijo es en la terminología platónica "un esclavo ignorante". La conquista del verdadero conocimiento exige madurar ideas, moldear los pensamientos y per- feccionar el discurso, es una tarea lenta, en la cual no se aceptan las improvisaciones.

Una de las crisis de la sociedad actual es tener demasiada información que no se asimila, esto lleva a que el diálogo se torne polémico al buscar respuestas rápidas, inmediatas, promoviendo opiniones y no el saber fundamentado. Lacan en los principios de la cura dice que "hay un tiempo interior que no es psicológico, es decir, que no consiste en que el sujeto sea lento o rápido, sino que todo orden de complejidad requiere un determinado tiempo para engendrar un sólido conocimiento".

\section{La diferencia entre la doxa y el episteme}

El conocimiento común no es metódico ni crítico, se queda en la superficie, en la sugestión de los sentidos, tomando los datos de la percepción como si fuera la realidad misma; los juicios que formula expresan menos opiniones. Aceptar que las verdades científicas son relativas no significa afirmar que la ciencia no tenga como meta la razón, la búsqueda de la verdad; como esta no significa poseerla, el investigador, a pesar de haber realizado su trabajo con todo rigor metodológico, honestidad y crítica, debe estar conciente que su verdad nunca puede aspirar al saber absoluto, puesto que siempre puede resultar otra verdad mejor fundamentada $u$ otras respuestas más apropiadas para resolver el problema que está enfrentando.

\section{Analfabetismo cientifico}

Blanca Inés Prada dice que los docentes universitarios en la actualidad: 1) Ignoran el dominio de un idioma extranjero y con dificultad llegan al mundo intelectual internacional. 2) Se lee muy poca literatura científica (menos que la literatura general). La pobreza en los hábitos de lectura de los docentes no promueve la pasión por el libro en el estudiante, la era de las fotocopias sin contexto se ha impuesto, y da como resultado la formación de profesionales mediocres, con muy débil formación científica y ética para lograr la interiorización de valores fundamentales para la convivencia democrática. 3) En Colombia la ciencia tiene muy poco espacio y aún los que han pasado por la universidad carecen de formación cien- 
tífica, esto es apropiación de conocimientos fundamentales, porque no han recibido una formación que les permita entender el desarrollo científico, ni siquiera dentro de la propia profesión. 4) Tienen desprecio por la profesión docente investigativa. El docente investigador necesita gozar de un mínimo de comodidades más allá de lograr sobrevivir, espera ante todo el reconocimiento social que le ayude desde el punto de vista moral a su tarea; requiere además: a) asignación de tiempo para preparar sus clases (tiempo real, coherente con las características de las temáticas propuestas, la búsqueda bibliográfica, la producción de material didáctico y audiovisual, etc.); b) tiempo para escribir documentos, redactar informes, participar en seminarios nacionales e internacionales, dialogar con sus estudiantes (y sus propios pares) y para corregir trabajos y tareas; c) el tiempo es poco valorado y casi menospreciado, incluso por los mismos colegas por falta de respeto en el escenario académico y consideración, para evitar comportarse como un comerciante de saberes. Kant dice " la honradez hace que uno reconozca sus errores y no encubra sus puntos flacos. Quien carece de ella maneja los productos del entendimiento como el comerciante sus mercancías, ocultando los puntos flacos y engañando al público en general". 5) Este desprecio por la labor docente y la investigativa tiene que ver también con el que se tiene en nuestra sociedad por el desarrollo teórico que contribuya a resolver problemáticas locales. K. Poper dice "los temas científicos son enunciados universalmente; son como todas las representaciones, sistemas de signos y símbolos... los temas son redes que lanzamos para apresar aquello que llamamos el mundo: para racionalizarlo, explicarlo y dominarlo, y tratamos de que la malla sea cada vez mas fina".

\section{La formación profesional en enfermería}

Cuando pienso en la formación universitaria y en particular de enfermeros, algunas de las preguntas que me inquietan son: ¿Es la universidad realmente un espacio de formación de profesionales? ¿Qué nos significa a los docentes la idea de formar profesionales? ¿Quizás la adquisición de conocimientos técnicos y científicos? ¿El dominio de temas culturales, académicos e investigativos? Mejor aún, capacitación en un área que para nuestro caso es ciencias de la salud. ¿Dominio del conocimiento de la disciplina de enfermería?

Desde mi formación en pregrado he concebido la formación profesional como ese espacio en el cual se me permitió, cultivó y potenció la integralidad de mi esencia humana desde la dignificación del SER.

\section{¿Qué define a un profesional?}

Ya en 1910 Abraham Flexner identificó varias características que aun hoy son válidas e importantes para definir un profesional: conocimiento, especialización, responsabilidad intelectual e individual y conciencia de grupo bien desarrollada. Después, varios autores de diversos campos han presentado otras perspectivas y reforzado las enunciadas por Flexner, con elementos como: autonomía, investigación, colegialidad (comprende identidad profesional, colegaje, es decir respeto en la interrelación con pares, agremiación y empoderamiento profesional), y colaboración e innovación (creatividad, recursividad). Integraré estos elementos que definen a un profesional en los tres que abordamos desde la formación, como son: ser, conocer y el saber hacer.

Es necesario recordar que la enseñanza en enfermería está fundamentada en el modelo militar y religioso por lo cual es autocrático, transmisionista, mecanicista y tradicionalista, por tanto hay que trasformar el paradigma educativo mas allá de las corrientes de mercado que transforman los nombres pero que en esencia continúan igual de rígidos y repetitivos, es necesario sensibilizar y formar a los docentes en el modelo pedagógico que promueve la Fundación Universitaria de Ciencias de la Salud, de la escuela activa y el aprendizaje significativo.

Es por eso que no podemos bajo ninguna razón olvidar que la labor académica universitaria debe jalonar los procesos sociales, políticos, económicos de la nación y que somos responsables por acción o por omisión de la situación actual de la salud y de la educación, al mantenernos en un aislamiento de los escenarios donde se toman las decisiones. Hemos dejado espacios vitales conquistados por nuestros maestros, espacios que fueron políticos, económicos, de liderazgo e identidad profesional. La invitación es a compartir esta disertación transversalizada por el liderazgo positivo, la identidad profesional y social 


\section{ELEMENTOS}

Actitud=ser: deseo de cuidar y capacidad para hacerlo

\section{DEFINICIÓN}

DESEO: implica mantener la atención centrada en lo que es mejor para el sujeto de cuidado, respetar valores, creencias, estar dispuesto a apoyarlos, a pesar de que sus problemas empeoren; además mantener siempre un estilo de vida saludable que permita ayudar a los otros y trabajar intensamente para que estas condiciones se conviertan en hábitos.

CAPACIDAD: contiene y requiere dos dimensiones específicas:

1. Comprenderse a sí mismo, implica aprender sobre las propias tendencias, reacciones, hábitos, los cuales cambian a medida que la persona madura y afronta diferentes situaciones; la capacidad requiere el compromiso de aprender sobre sí mismo y reconocer cómo los propios valores y el marco de referencia puede influir en el pensamiento y la capacidad para comprender a los demás, facilitando un pensamiento cada vez más objetivo.

2. Comprender a los demás, implica aprender a escuchar empáticamente; significa conectarse con los sentimientos del otro, identificarse con sus luchas, con sus frustraciones, con sus necesidades y deseos, y después desprenderse de esos sentimientos y recuperar el propio marco de referencia. Esta capacidad permite identificar necesidades, prioridades y hallar objetivos comunes.

Estos elementos fundamentalmente son brindados $y / 0$ fortalecidos mediante la salud mental y la ética.

Los valores indispensables para cultivar son: la constancia, atención, exactitud, precisión, responsabilidad, respeto, prontitud, vigilancia y puntualidad.

Los principios bioéticos que se deben cultivar son: beneficencia, autonomía y justicia.

\section{Conocimiento=saber:}

qué hacer y por qué hacerlo
El conocimiento es muy diverso y el enfermero debe esforzarse por actualizarse en forma permanente para actuar de manera segura, eficiente y oportuna de acuerdo con la información científica existente, esa base de conocimientos incluye tres áreas:

1. Las ciencias básicas: matemáticas, química, física, biología, bioquímica, anatomía, fisiología, microbiología, farmacología, fisiopatología, etc.

2. Las humanísticas: comunicación, legislación, ética, sociología, antropología, filosofía, psicología, contexto social, economía, política, artes, deportes, etc.

3. Las específicas profesionales: donde encontramos todas las áreas de cuidado de la salud y de la vida por ciclo vital o como lo tenga diseñado cada facultad o programa.

Los valores indispensables para cultivar son: dedicación, disciplina, aplicación, lectura, enseñanza, sistematización, corrección, respeto, responsabilidad, estímulo, compromiso, motivación, aprendizaje.

Los principios bioéticos que se deben cultivar son: beneficencia, autonomía y justicia. 
Habilidad=saber hacer: cómo hacerlo
Implica el desarrollo de habilidades:

1. Manuales: las cuales se requieren para desarrollar en forma eficiente los procedimientos técnicos y se va adquiriendo en el laboratorio y en la experiencia clínica.

2. Intelectuales: son indispensables para resolver problemas y tomar decisiones; el pensamiento crítico en enfermería supone uno deliberado y dirigido al logro de los objetivos; pretende emitir juicios basados en pruebas no en conjeturas (conceptos, no opiniones) y se fundamenta en los principios de la ciencia y el método científico.

3. Interpersonales: se requieren para establecer relaciones positivas y productivas; la incapacidad de hacerlo impide el conocimiento de los hechos reales, la comprensión de la esencia de los problemas y el poder obtener ayuda de los demás o ser miembro efectivo del equipo de cuidadores y de la salud, ya que hace viable el trabajo intergremial, interdisciplinar, transdisciplinar.

Los valores indispensables para cultivar son: amabilidad, respeto, benevolencia, prudencia, fraternidad, tolerancia, aprecio, consideración, estimación, gratitud, solidaridad.

Los principios bioéticos que se deben cultivar son: beneficencia, autonomía y justicia. fuerte, y la toma de decisiones políticas para lograr los tres elementos en los que creo que se debe centrar la universidad que no son nuevos, no los estoy descubriendo yo, sino reconquistándome, recreándome en el trascendente sentido que representan cada día más.

Recreación y reconquista que integra, en el proceso de formación de los profesionales, prácticas pedagógicas sensibles, coherentes y dinamistas como las planteadas por Platón en sus disertaciones pedagógicas, donde encantemos-enamoremos a los estudiantes con el ser de enfermería para que se comprometan con el saber y el querer hacer, de esa manera sueño que la universidad como expresión genérica, recobre el papel formador de profesionales útiles para la sociedad más allá del desempeño específico de una tarea u oficio laboral.

Para finalizar, retomando unas palabras del ya fallecido Héctor Abad Gómez sobre el desempeño docente universitario, las dejo en el cierre para su reflexión personal y colectiva, colegas y estudiantes de enfermería, ya que es indiscutible que el conocimiento y desarrollo profesional se debe ver reflejado en actos humanos de cuidado, de otra manera estaremos como universidades en- trando al proceso tayloriano de entrega de títulos más no de formación de profesionales:

"El mero conocimiento no es sabiduría. La sabiduría sola tampoco basta. Son necesarias la sabiduría y la bondad para enseñar y gobernar a los hombres. Aunque podríamos decir que todo hombre sabio, si verdaderamente lo es, tiene también que ser bueno. Porque la sabiduría y la bondad son dos cosas intimamente entrelazadas. Lo que deberíamos hacer los que fuimos alguna vez maestros sin antes ser sabios, es pedirles humildemente perdón a nuestros discipulos por el mal que les hicimos".

\section{Lecturas recomendadas}

- Abad Gómez H. Manual de tolerancia. 4a ed. Medellín: Editorial Universidad de Antioquia; 1996.

- Bauman, A. Entornos de práctica favorables: lugares de trabajo de calidad atención de calidad al paciente. Carpeta de herramientas de información y acción. Ginebra: Consejo Internacional de Enfermeras CIEK; 2007. 
- Cortina, Adela. Ética de la sociedad civil : Madrid, España: Anaya, 1994.

- Cortina A. La educación del hombre y del ciudadano. Rev. Iberoam. Educ [serie en Internet] 1995 [citado 28 ene 2008]; 7 [aprox. 10 p.]. Disponible en: http:// www.rieoei.org/oeivirt/rie07a02.pdf

- Cortina A. Éticas del deber y éticas de la felicidad [monografía en Internet]. Madrid, España: Fundación Xavier Zubirí; 1996 [citado 28 ene 2008]. Disponible en: http://www.zubiri.net/bol5.html

- Delors J, et al.. La educación encierra un tesoro: informe a la UNESCO de la Comisión Internacional sobre la Educación para el siglo XXI. Paris : UNESCO; 1996.

- Gutiérrez de Reales E. El carácter de la enfermera que cuida. Revista Kaana- úai. 2007; 2(1): 8-14.
- Hernández Arriaga JL. Bioética General. Bogotá : Manual Moderno; 2002.

- Ibáñez LE, Sarmiento de Peñaranda LV. El Proceso de enfermería. 1a ed. Bucaramanga: Editorial Universidad Industrial de Santander; 2003.

- Molina P. Cuidado y enfermería. Revista Kaana-úai, 2007; 2(1): $15-17$.

- Moore GE. Ética. Bogotá: Labor; 2000.

- Murrain Knudson E. Enfermería de cara al siglo XXI: hacia la redefinición de la formación y la práctica. Revista Kaana-úai. 2007; 2(1): 36-39.

- Prada Márquez BI. Epistemología, universidad, ética y valores, ensayos. Bucaramanga: Editorial Universidad Industrial de Santander; 1995.

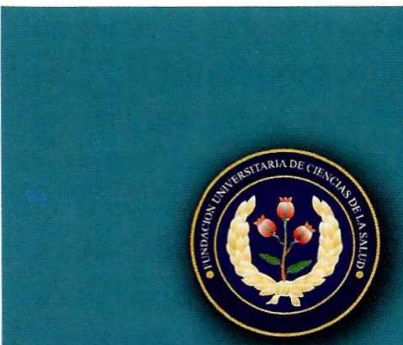

\section{FUNDACIÓN UNIVERSITARIA DE CIENCIAS DE LA SALUD HOSPITAL DE SAN JOSÉ}

Personeria Juridica: 10917 01-12-1976 Resolución Ministerio de Educación Nacional No. 0125

Facultad de Instrumentación Quirúrgica Código lcfes 270246100281100111100

Título Otorgado Instrumentador Quirúrgico
Oficina de admisiones: Carrera 19 No 8 A-32 Edificio docente P. 1 PBX: 3538100 Ext: $3615 / 3613$ Fax: 3614

E-mail: instrumentacion@fucsalud.edu.co

http://www.fucsalud.edu.co

\begin{tabular}{|c|c|c|c|c|c|c|c|}
\hline $\begin{array}{l}\text { I SEMESTRE } \\
\text { *Biología } \\
\text { *Morfofisiologíal } \\
\text { *Física } \\
\text { *Química } \\
\text { *Introducción a la } \\
\text { instrumentación } \\
\text { *Comunicación } \\
\text { oral y escrita } \\
\text { *Informática }\end{array}$ & $\begin{array}{l}\text { II SEMESTRE } \\
\text { *Microbiología } \\
\text { *Morfofisiología II } \\
\text { *Esterilización } \\
\text { *Bioquímica } \\
\text { "Técnicas } \\
\text { quirúrgicas I } \\
\text { *Técnicas de } \\
\text { Instrumentación I } \\
\text { *Sociología }\end{array}$ & $\begin{array}{l}\text { III SEMESTRE } \\
\text { *Patología I } \\
\text { *Cuidados básicos } \\
\text { en salud } \\
\text { *Farmacología } \\
\text { *Anestesia } \\
\text { *Técnicas } \\
\text { quirúrgicas II } \\
\text { *Técnicas de } \\
\text { Instrumentación II } \\
\text { *Ingles I } \\
\text { *Psicología }\end{array}$ & $\begin{array}{l}\text { IV SEMESTRE } \\
\text { *Patología II } \\
\text { *Epidemiología } \\
\text { *Técnicas } \\
\text { quirúrgicas III } \\
\text { *Técnicas de } \\
\text { Instrumentación III } \\
\text { *Ingles II } \\
\text { *Constitución } \\
\text { política y } \\
\text { derecho en salud } \\
\text { *Ètica General } \\
\text { *Administración } \\
\text { general } \\
\text { *Investigación I }\end{array}$ & $\begin{array}{l}\text { V SEMESTRE } \\
\text { *Práctical } \\
\text { *Bioética } \\
\text { *Administración } \\
\text { hospitalaria } \\
\text { *Investigación II }\end{array}$ & $\begin{array}{l}\text { VI SEMESTRE } \\
\text { *Práctica II } \\
\text { *Didáctica } \\
\text { *Proyecto de } \\
\text { investigación }\end{array}$ & $\begin{array}{l}\text { VII SEMESTRE } \\
\text { *Práctica lil } \\
\text { *Mercadeo } \\
\text { *Seminario de } \\
\text { investigación }\end{array}$ & $\begin{array}{l}\text { VIII SEMESTRE } \\
\text { *Práctica IV }\end{array}$ \\
\hline
\end{tabular}

